\title{
EDUCANDO PARA QUÉ Y PARA QUIÉN. EL CAMBIO DE PARADIGMA QUE EL DESARROLLO SOSTENIBLE DEMANDA A LA EDUCACIÓN SUPERIOR ${ }^{1}$
}

\author{
JoRdi SEGALÀs CORAL \\ Director del Instituto Universitario de Investigación en Ciencia y Tecnologías de la Sostenibilidad
}

DOI: $10.1387 /$ lan-harremanak.15389

\section{ABSTRACT}

La sociedad tal como está organizada actualmente no tiene futuro, es insostenible; tanto ambiental, social, como económicamente. Estamos en una situación con un gran incremento de demandas al ecosistema y una disminución de recursos disponibles que conlleva a la aparición de conflictos sociales. Esto es debido a un paradigma económico neoliberal que prioriza el crecimiento a cualquier precio sin tener en cuenta los límites finitos del planeta y que incrementa las diferencias entre los ganadores y perdedores del sistema. Incluso la Unión Europea acuña el concepto de crecimiento sostenible, que per se es un oximoron en un sistema finito como el nuestro.

Es necesario un cambio de paradigma en tres ejes: primero, el marco en que nos organizamos desde el punto de vista cultural (valores y principios que justifiquen necesidades: suficiencia), estructural (estructura que permite aplicar dichos valores y principios) y tecnológico (medios para satisfacer las necesidades); segundo, los saltos de innovación (uso óptimo, mejora de procesos y productos y renovación basada en

\footnotetext{
${ }^{1}$ Resumen de la ponencia presentada en el II Congreso estatal de centros universitarios de relaciones laborales y ciencias del trabajo, organizado por la UPV/EHU, y celebrado en Bilbao los días 18 y 19 de junio de 2015.
} 
necesidades); y tercero, la transdisciplinariedad, en relación a los actores implicados o afectados por cualquier decisión socio-politica-tecnológica.

Este cambio de paradigma social requiere un cambio de paradigma educativo a tres niveles: primero, definir un nuevo paradigma educativo que incluya en su visión $y$ misión los valores de la sostenibilidad; segundo, a nivel de organización y gestión, definiendo cómo se organiza y gestiona la universidad en todos sus ámbitos de influencia (educación, investigación, gestión); y, finalmente, en las metodologias de aprendizaje.

Palabras clave: educación superior, educación sostenible, sostenibilidad.

Society as it is currently organized does not have future, It is unsustainable; environmental, social, and economically. We are in a situation with a large increase in demands on the ecosystem and a decrease in available resources that leads to the emergence of social conflicts. This is due to the neoliberal economic paradigm that prioritizes growth at any price without taking into account the finite limits of the planet and increases the gap between the winners and losers of the system. Even the European Union coined the concept of sustainable growth, which per se is an oxymoron in a finite system like ours.

A paradigm shift is needed in three areas; first, the framework in which we are organized from the cultural point of view (values and principles that justify needs: sufficiency), structural (structure that allows to apply those values and principles) and technological (means to meet the needs); second, innovation breaks (optimal use, process and product improvement $t$ and renovation based on needsn); and third, transdisciplinarity in relation to the stakeholders involved or affected by any sociopolitical and technological decision.

This change in the social paradigm requires a change of the educational paradigm at three levels: first, define a new educational paradigm to have in its vision and mission the sustainability values. Second level is the organization and management, defining how the educational system is organized and managed by the university in all its areas of influence (education, research, management); and finally, learning methodologies.

Key Words: higher education, sustainable education, sustainability. 


\section{Introducción}

Vamos a analizar el rol de la educación superior para promover el desarrollo sostenible o un futuro sostenible. El contenido está estructurado en torno a los tres ámbitos siguientes: primero, cuál es el reto que tenemos como sociedad; segundo, cuál es el cambio de paradigma que se requiere y, tercero, relacionándolo con el sistema educativo, cuál es el cambio del paradigma en el sector educativo.

\section{El reto de la sostenibilidad}

Empezaremos con los retos de sostenibilidad que tiene nuestra sociedad actualmente. Vamos a definir un marco de referencia entorno al concepto de sostenibilidad, dado que cuando se habla de sostenibilidad hay múltiples acepciones y suele suceder lo mismo que les sucedía a los actores principales de la leyenda hindú de los ciegos y el elefante (ver figura 1), donde siete personas cie-

Figura 1

Los ciegos y el elefante

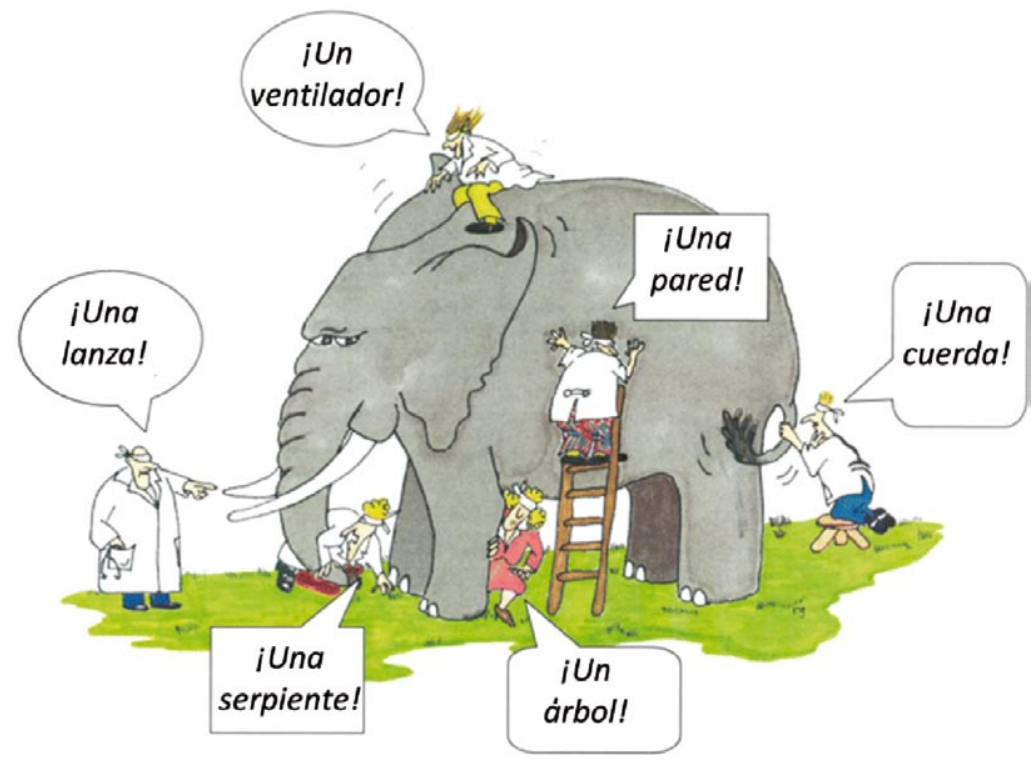

https://es.wikipedia.org/wiki/Los_ciegos_y_el_elefante 
gas se acercan a un elefante y, en función de qué parte del elefante tocan, identifican que se trata de una cosa o se trata de otra. Con la sostenibilidad pasa algo similar. En función de nuestro histórico, en función de nuestros intereses, en función de qué es lo que queremos, entendemos la sostenibilidad de una manera o de otra. Y por ahí empieza un primer problema, en la epistemología del concepto de sostenibilidad. Vamos a intentar inicialmente poner en común el significado de sostenibilidad para que todos entendamos de qué estamos hablando.

Básicamente hay dos marcos para definir lo que es la sostenibilidad. La triple bottom line que define los tres pilares de la sostenibilidad: social, económico y ambiental. Otro marco de trabajo muy extendido son los capitales de la sostenibilidad, con lo cual se le da un valor económico a las partes social, natural y la parte económica, con lo cual podemos ya empezar a comparar entre cada uno de estos capitales y podemos empezar a transferir moneda entre un capital y otro. Y lo que se intentaría con la sostenibilidad es que todos los capitales estuviesen representados de una manera equitativa y que cualquier acción que afecte al sistema incrementase los tres capitales. Pero nos encontramos que, actualmente la mayoría de sociedades occidentales tenemos otra visión del mundo: damos como leyes inevitables las leyes económicas (capital económico), las leyes del mercado. A posteriori el medio ambiente (capital ambiental) es utilizado para cubrir las demandas de estas leyes económicas y que básicamente proporciona recursos y energía y absorbe los residuos y las emisiones. Y aparte tenemos la sociedad (capital social), que se tiene que adaptar a estas leyes del mercado (figura 2).

Figura 2

Visión del mundo actual. Importancia relativa

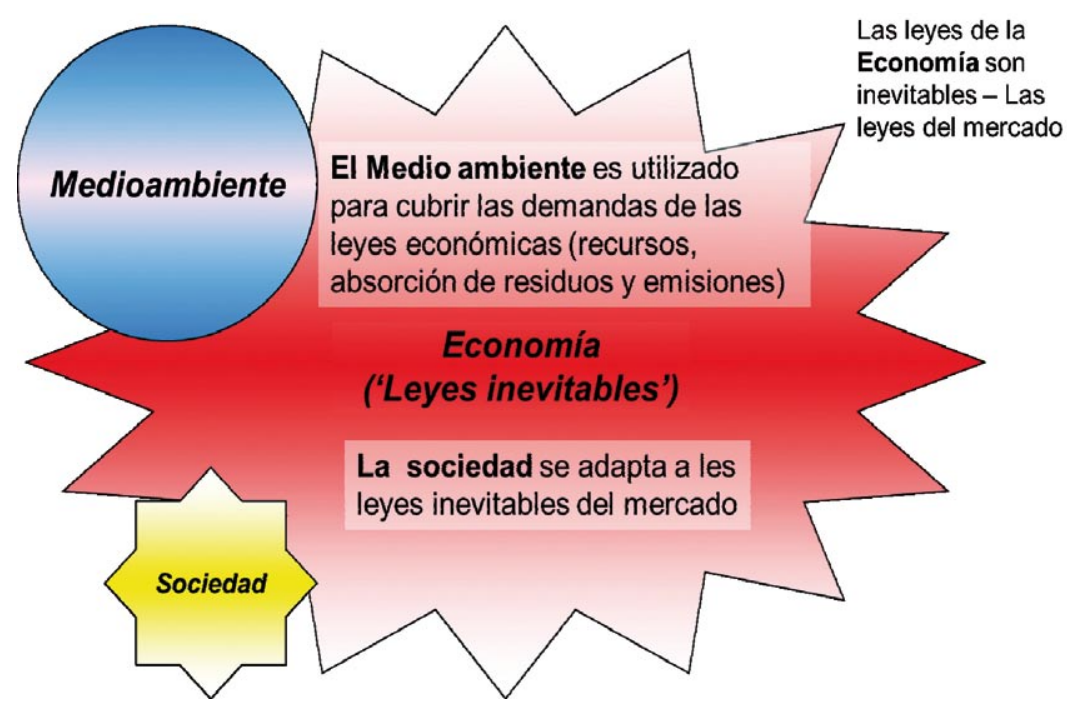


Sin embargo, lo que sí que está demostrado científicamente es que las leyes que son inevitables son las leyes ambientales (capital ambiental): primera, segunda ley de la termodinámica, etcétera. Este medio ambiente soporta y ayuda a soportar a la sociedad (capital social) con todas las mezclas de leyes, culturas, etcétera. Y como sociedad en unos momentos determinados hemos ido diseńando una manera de organizarnos basada en parte en una economía (capital económico) que ha ido cambiando a lo largo de nuestra historia. Actualmente tenemos la economía neoliberal, que es la que decide las leyes del mercado actual y que se pretende que no podemos cambiar, y que nos tenemos que adaptar a ellas. Pero lo que realmente sucede es que esta economía que hemos inventado con los indicadores que vamos a ver ya no nos sirve, es decir, las leyes inevitables son las leyes naturales y no las leyes del mercado (que hemos inventado nosotros), y que simplemente son una manera de organizarnos. Pongo esto en énfasis para tener en cuenta que, si este sistema no nos funciona, tendremos que hacer algo para cambiarlo. La figura 3 muestra un modelo sostenible, donde el medio ambiente soporta la sociedad, que se organiza de una determinada manera con unas leyes económicas determinadas.

Figura 3

Visión real del mundo

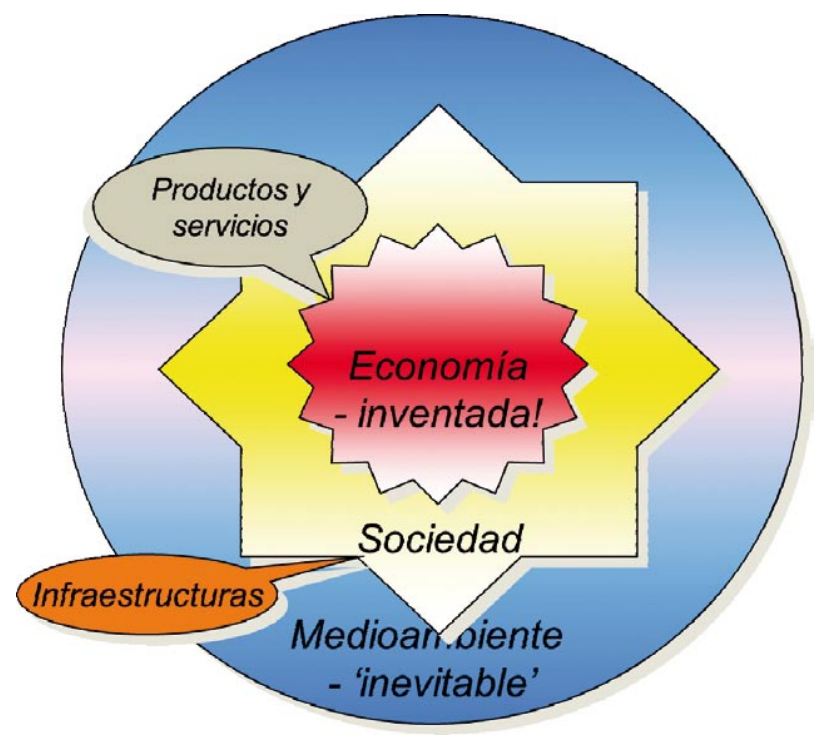


Porque, al final, se trata de que como especie tenemos unos recursos ambientales y tenemos unos objetivos (figura 4). Y entiendo que todo el mundo coincidirá, o mayoritariamente coincidiremos, en que los objetivos que tenemos como sociedad es tener una vida feliz, larga y satisfactoria... Y para pasar de unos recursos finitos a conseguir el objetivo antes mencionado están los medios. Como medios actualmente nos encontramos con la comunidad, la tecnología, la sanidad, la economía, los valores, la familia, etcétera. Pero estos son solo medios, y actualmente para la mayor parte de nuestra sociedad parece que la mejora económica no es un medio sino el objetivo final.

\section{Figura 4}

Recursos, medios y objetivos de la sociedad humana

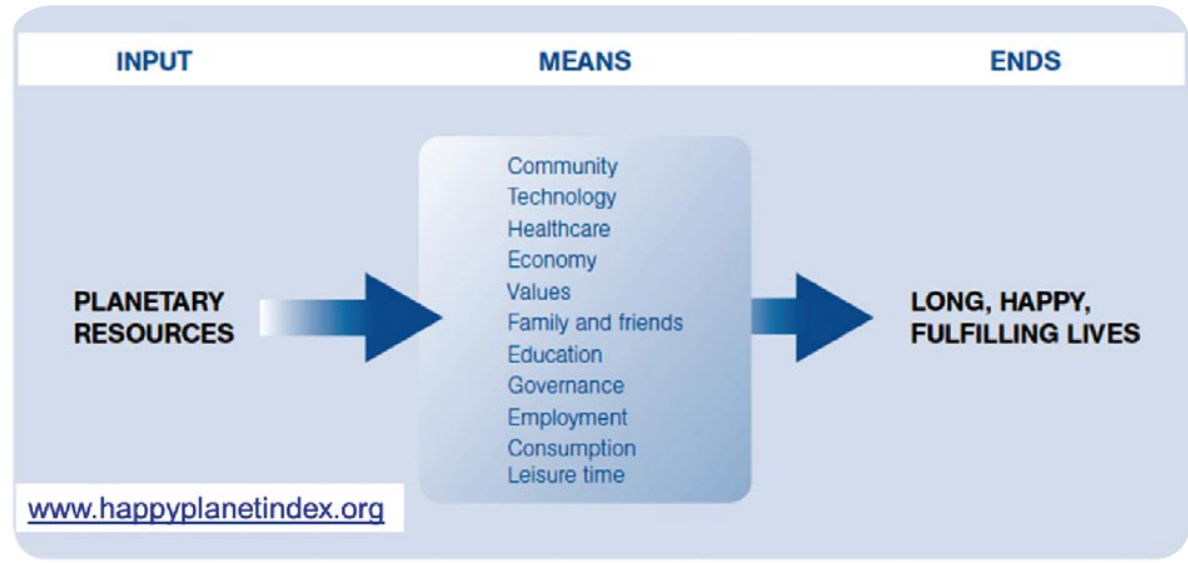

Es decir, replanteando este reto sería: ¿cómo podemos hacer que $10.000 \mathrm{mi}-$ llones de personas sean felices en este planeta? Y esto nunca había pasado porque nunca habíamos llegado a estos valores de población y de consumo, con lo cual es un reto mayúsculo, que conlleva solucionar múltiples problemas a todos los niveles (figura 5): a nivel económico, a nivel de recursos naturales, de la salud ambiental, energía, de suministro de alimentación, de lo que sería incremento de población, transporte, etcétera, etcétera. 
Figura 5

Problemas globales más importantes

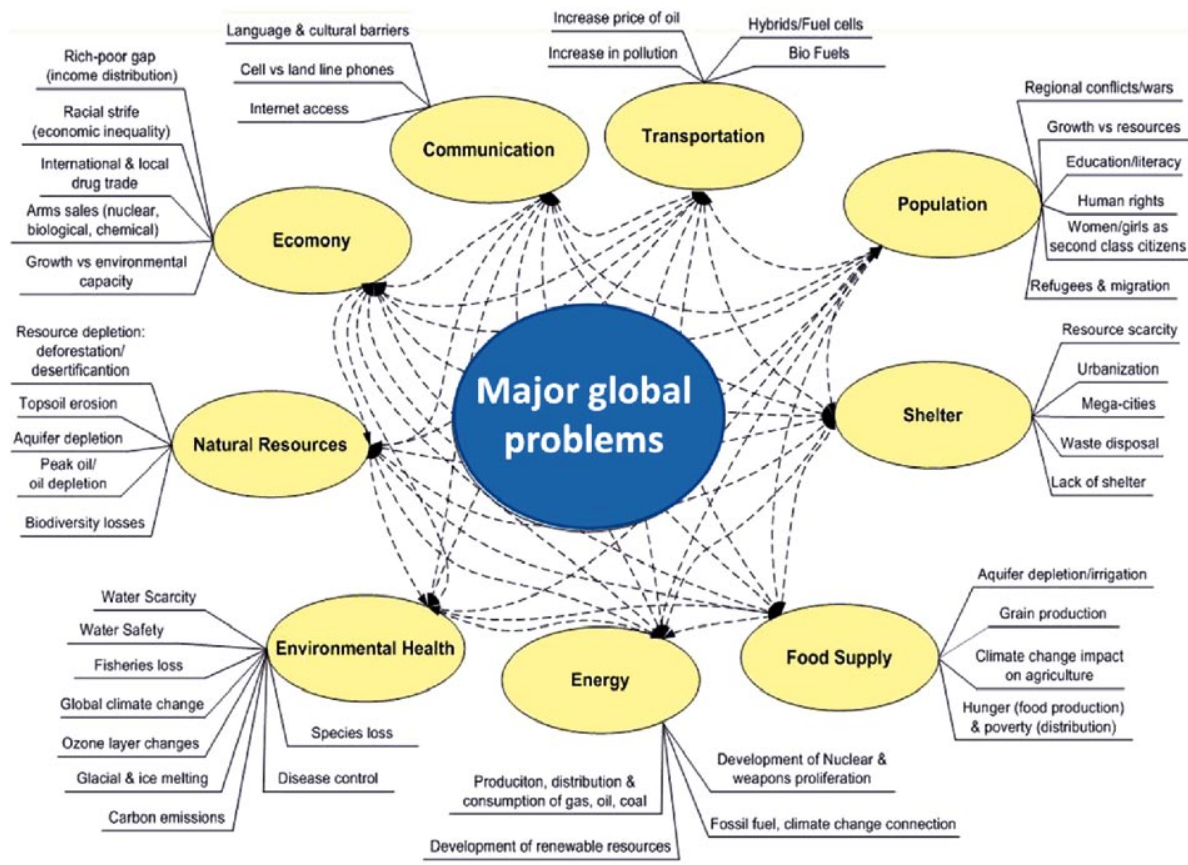

¿Y cómo nos va respecto a estos retos? Hay muchos informes: el estado del mundo, The Living Planet Report, el informe del desarrollo humano. A nivel europeo tenemos el Eurostar que publica indicadores de sostenibilidad, el IPCC, que es el que analiza todo el cambio climático con miles de científicos trabajando en esos informes. Todos esos informes se editan anualmente. ¿Y qué nos indican estos informes? El resultado lo podemos expresar con la metáfora de un embudo (figura 6). 
Figura 6

Metáfora del embudo

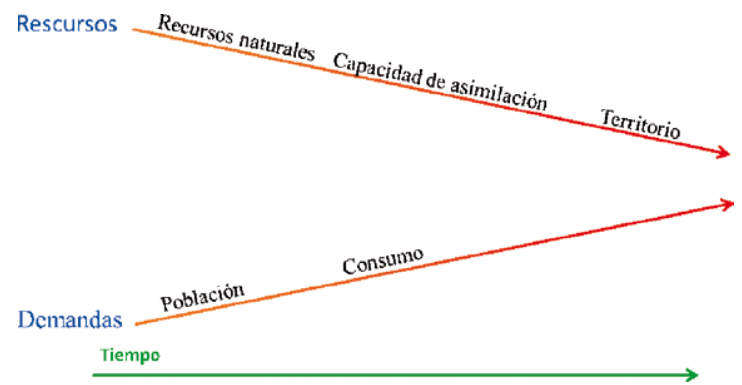

En el eje horizontal tenemos la evolución. Y lo que ha ido sucediendo es que a lo largo del tiempo han aumentado las demandas por parte de nuestra sociedad y han ido disminuyendo los recursos. ¿Qué demandas? La primera es la población: la población ha ido aumentando en los últimos ańos... Ha ido aumentando siempre, pero especialmente los últimos 40 años, siendo actualmente de en torno a los 7.500 millones y se prevé que en el 2100 llegue a los 10.000 millones.; y, además, con una la distribución muy dispar. La mayor parte de población está y estará en Asia, África... La segunda es el aumento del consumo. Que de por sé no es nocivo, siempre que sea como medio para llegar al objetivo final que comentábamos antes. La figura 7 muestra cómo ha ido aumentando el PIB en diferentes países del mundo:

Figura 7

Incremento PIB mundial

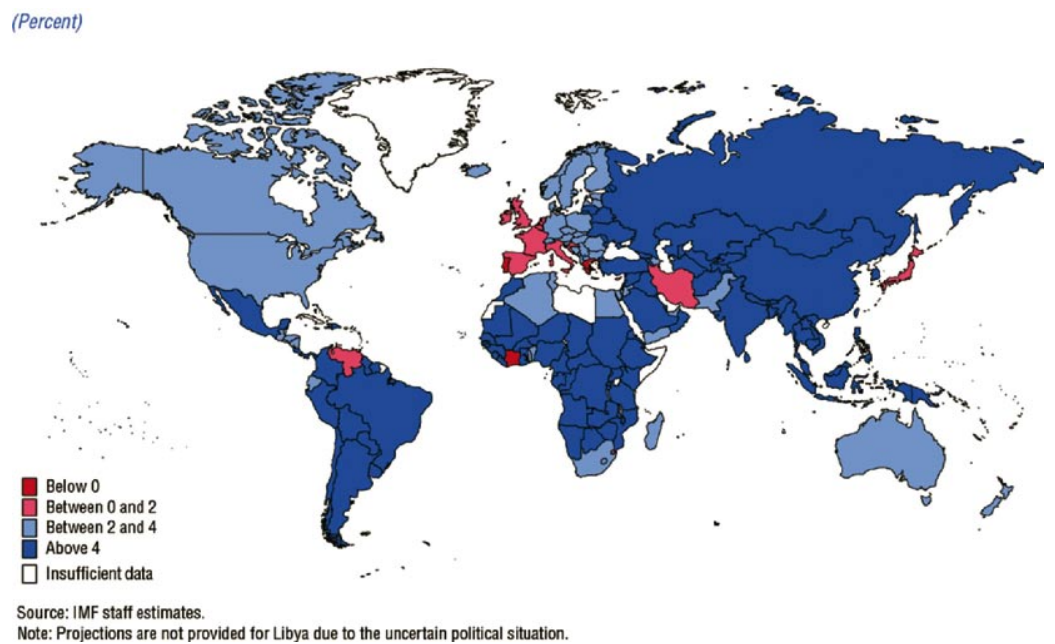


A nivel global ha ido aumentando el PIB y sigue aumentando año tras año. La zona de los BRICS es donde han aumentado más. Y el sistema se basa en el crecimiento, si no crecemos tenemos un problema, dado que si no crecemos y no consumimos, no podemos mantener los lugares de trabajo y se colapsa el sistema.

Y los recursos, ¿qué pasa con los recursos? Los recursos naturales simplemente han ido disminuyendo: un ejemplo típico es el tema del petróleo. Hay diferentes estudios que dicen que se acabará dentro de 30, 40, 50 años. Es igual: de aquí a 200 años no va a haber petróleo y nos tendremos que acostumbrar a vivir sin petróleo (figura 8 ).

Figura 8

Fin de la era del petróleo
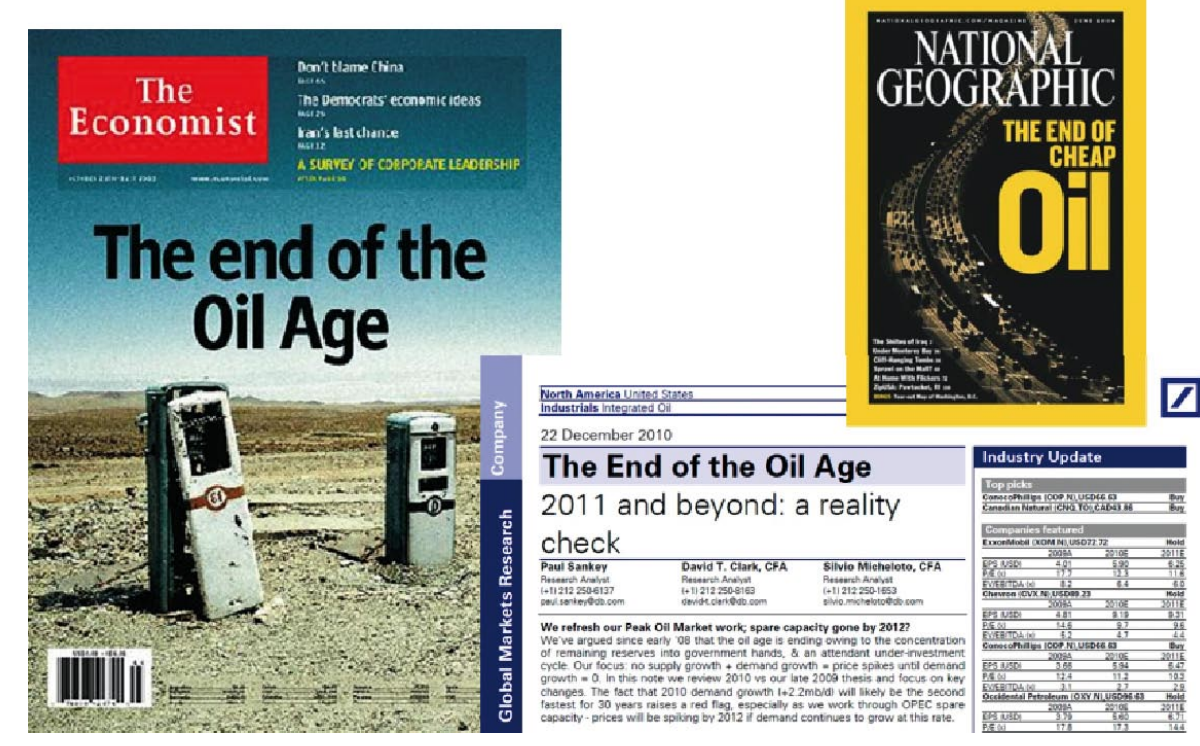

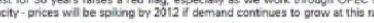

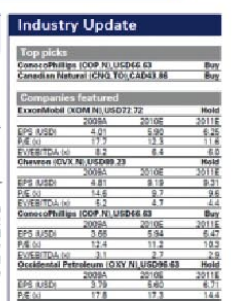

Y además estamos gastando el petróleo básicamente para producir energía, cuando el petróleo es imprescindible como fuente de materiales como, por ejemplo, de plásticos y si lo utilizamos para quemarlo, no podremos utilizarlo como material. Otro ejemplo es el fósforo (figura 9) que es básico para la agricultura: pues se prevé que haya un pico del fósforo en el 2033 y a partir de ahí va a disminuir la producción de fósforo. Con lo cual vamos a tener consecuencias para la producción de alimentos. 
Figura 9

Pico de producción de fósforo

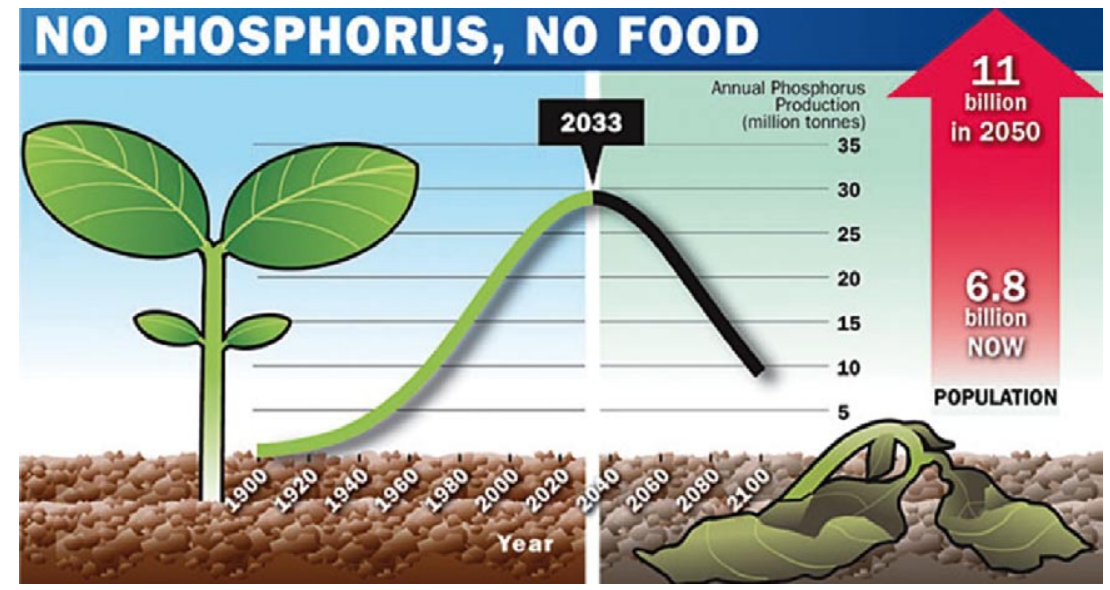

http://rock-phosphate.com/Research.html

La figura 10 muestra cómo ha ido evolucionando — datos hasta el 2007la extracción de materiales: de metales, de combustibles fósiles, biomasa. En 27 años ha aumentado la extracción un $60 \%$. Y sigue con esa tendencia. Si se compara con indicadores económicos, la figura 11 muestra como estos han ido evolucionando en 27 años (1980-2007).

Figura 10

Extracción global de recursos

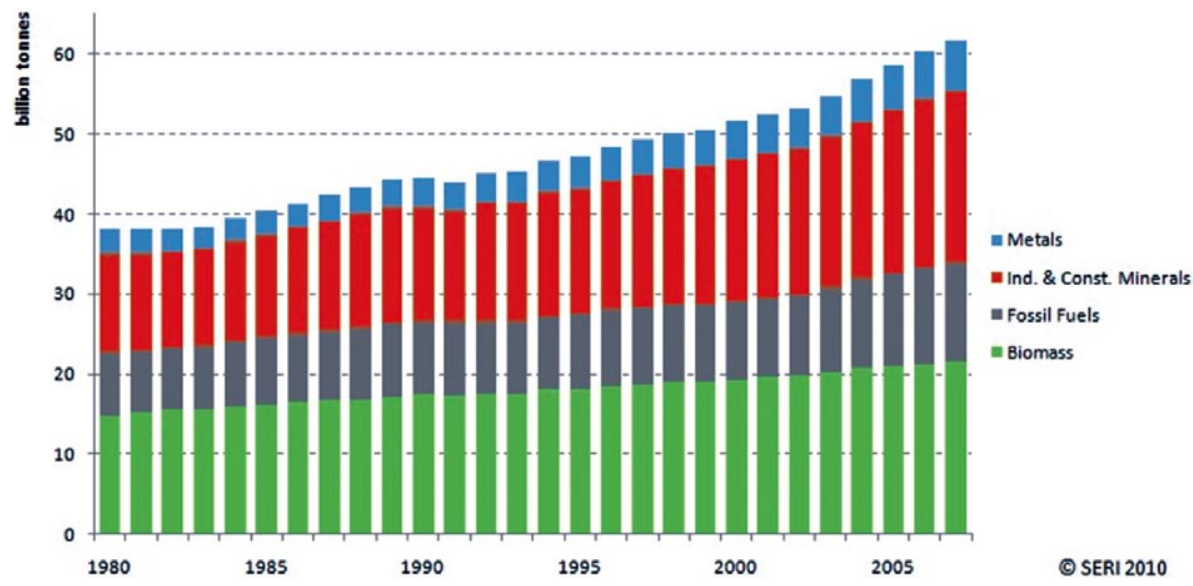


Figura 11

Tendencias en PIB, Extracción de recursos, Población e Intensidad material

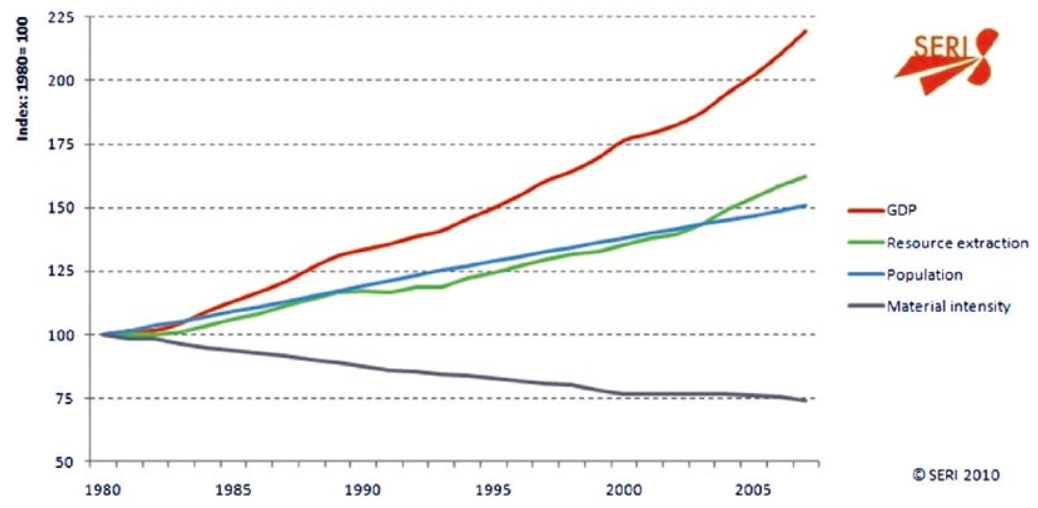

En 27 años el PIB ha aumentado un $120 \%$, la población ha aumentado un $50 \%$ y la extracción de recursos un $62 \%$. También hay una innovación tecnológica de la que hablaremos en un momento. Es la intensidad de materiales. Es decir, para aumentar una unidad de PIB cuántos recursos se requieren. Somos un $25 \%$ más eficiente en este sentido. Aun así, a pesar de ser un $25 \%$ más eficientes, hemos aumentado la extracción un $62 \%$.

También tenemos problemas respecto a la capacidad de asimilación. Caso paradigmático es el $\mathrm{CO}_{2}$. Actualmente ya estamos en más de 400 partes por millón de $\mathrm{CO}_{2}$ en la atmósfera. Lo cual tiene implicaciones en cuanto a incrementos de temperatura (figura 12). Los efectos que conlleva a nivel de producción de alimentos, a nivel de agua, a nivel de ecosistemas, a nivel de catástrofes climáticas. En función de cuánto se incrementa la temperatura promedio. 
Figura 12

Evolución de la concentración de $\mathrm{CO}_{2}$ atmosférico

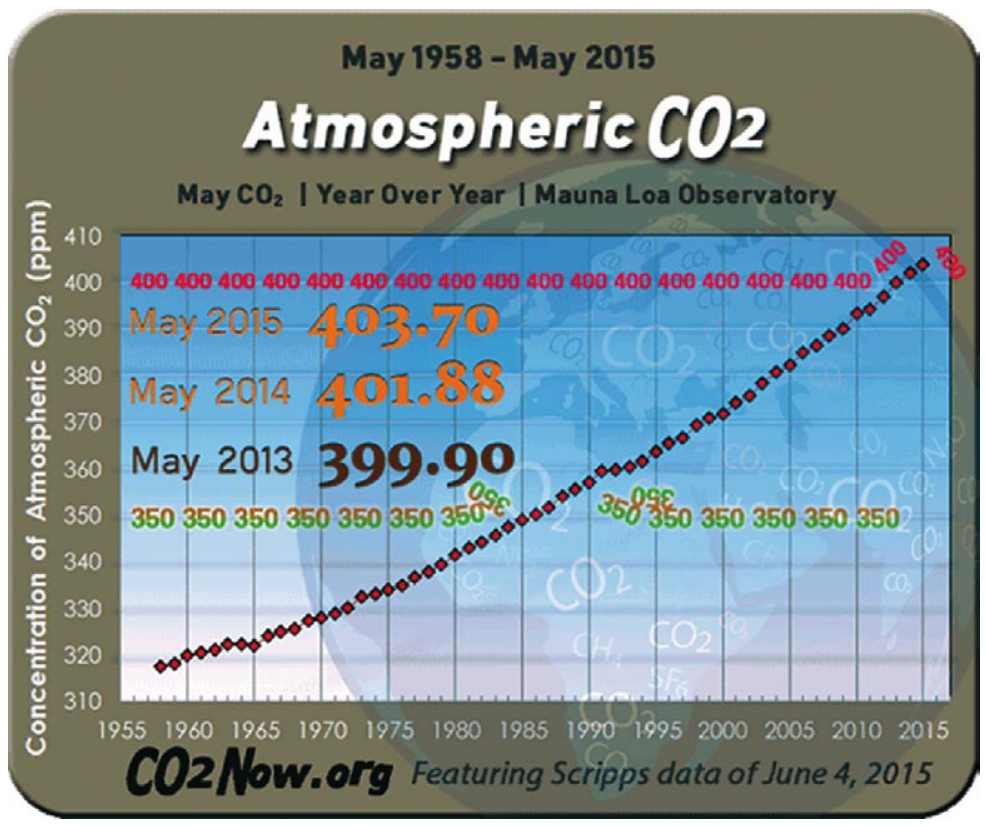

Figura 13

Impactos previstos en función de la magnitud del cambio climático

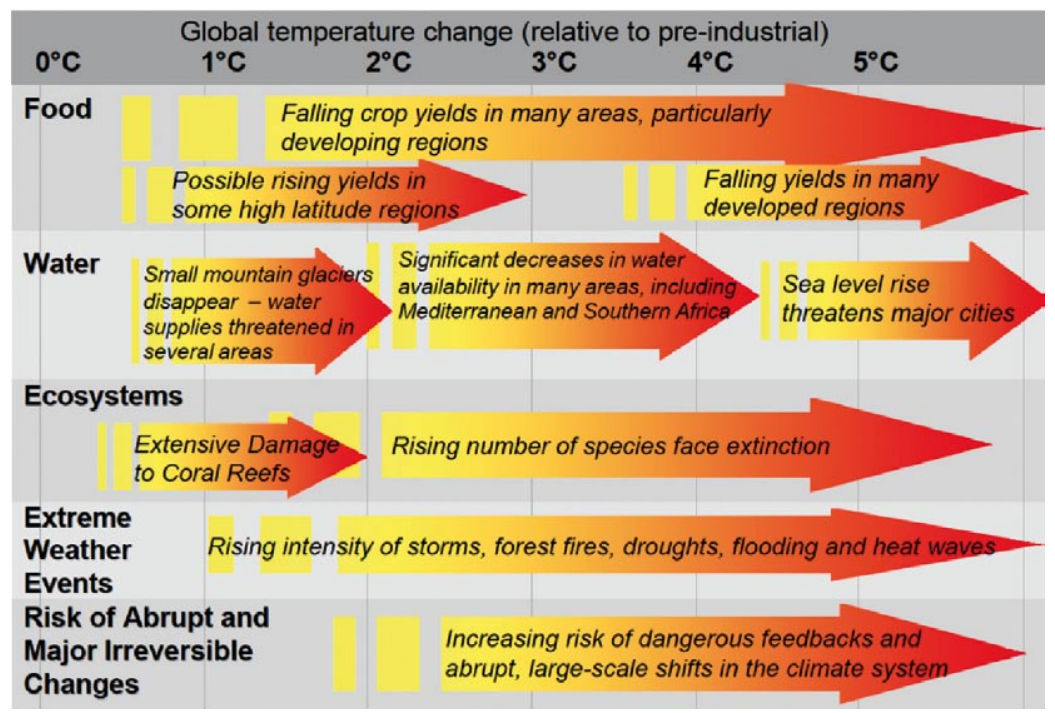


Otro problema asociado con el $\mathrm{CO}_{2}$ es la acidificación de los océanos. Se prevé que el $\mathrm{PH}$ de los océanos vaya disminuyendo, volviéndose más ácidos y variando toda la cadena trófica y disminuyendo la producción de pescado. Además es un bucle, cuanto más se acidifica menos capacidad tiene la absorción de $\mathrm{CO}_{2}$.

Resumiendo, la tendencia es, por un lado, un incremento de la demanda y, en paralelo, una disminución de la oferta (recursos). Y nuestra sociedad va chocando entre las dos paredes de este embudo (figura 14), y siempre que chocamos contra una pared tenemos un conflicto y nos tenemos que ir readaptando. Este espacio entre demanda y oferta cada vez es más estrecho y se incrementa la cantidad e intensidad de conflictos sociales.

Figura 14

Metáfora del embudo y cambios de paradigma

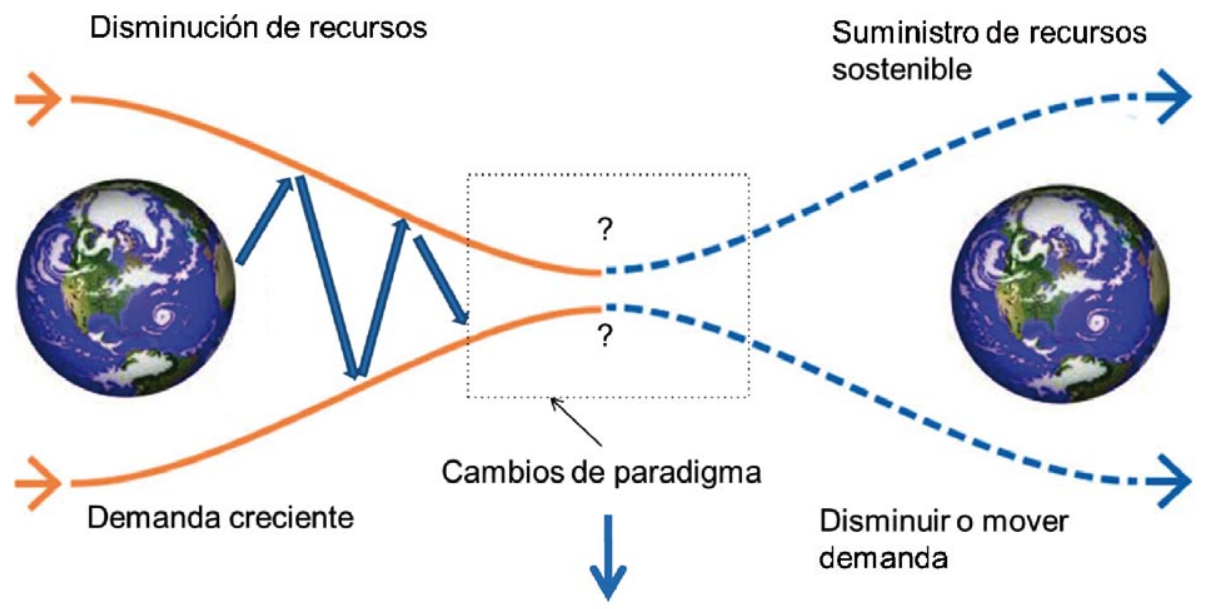

Cambios del paradigma Educativo

\section{Cambio de paradigma}

Si el sistema se colapsa, la solución pasa por un cambio de paradigma. ¿En qué dirección? En disminuir la demanda y que el suministro de recursos sea sostenible. ¿Cuál es una de las claves de este cambio de paradigma? El cambio del paradigma educativo, ¿para qué y para quién estamos educando? Si somos conscientes de que necesitamos cambiar el paradigma. Vamos a ver cómo podemos cambiar este paradigma. Y aquí hay los tecno-optimistas, que afirman: "Con la tecnología vamos a solucionar parte de estos problemas, porque vamos a ser muy 
eficientes. Por ejemplo: el coche eléctrico va a solucionar la movilidad». Cuando ya se sabe que no va a solucionar nada, simplemente va a desplazar el problema. Entonces vamos a evaluar qué rol puede jugar la tecnología y hasta dónde puede llegar. Para ello vamos a utilizar la ecuación IPAT que es: el impacto ambiental (I) es proporcional a la población (P) multiplicado por el consumo de cada persona (A), y multiplicado por el impacto de cada unidad de consumo que está relacionado con la tecnología (T) utilizada por unidad de consumo. Vamos a analizar cada uno de los parámetros de la ecuación por separado.

— Impacto ambiental (I): ¿cuál es el impacto ambiental que tenemos actualmente? Lo medimos con el concepto de huella ecológica. Huella ecológica es la cantidad de superficie que una sociedad necesita para vivir. Y dependerá de la población y cómo esta viva. Y tiene en cuenta la energía y los recursos que se consumen. Actualmente la disponibilidad per cápita que hay, está en torno de 1,7 hectáreas por persona y en cambio se están consumiendo 2,7 ha/persona. Es decir, consumimos 1.5 planetas y, si seguimos con esta tendencia, vamos a llegar dos, tres planetas.

- Población (P): en relación a la población, la tendencia es que vaya creciendo: 10.000.000.000 personas en 2100 .

- Consumo per cápita (A-Affluence, en inglés): para el consumo per cápita la tendencia es que vaya aumentando con los patrones económicos actuales. (Figura 7)

- Tecnología (T): cada vez somos más eficientes y disminuye la intensidad energética y la intensidad de materiales por unidad de consumo. Pero, ¿somos suficientemente eficientes para contrarrestar el aumento de la población y el aumento del consumo? Hagamos un pequeño ejercicio de proyección: imaginemos que queremos disminuir el impacto por ejemplo para el año 2050 un $50 \%$. Es decir, que en el año 2050 como población solo consumamos un planeta. ¿Cuál tendría que ser el avance tecnológico para conseguirlo teniendo en cuenta las tendencias de aumento de población y aumento de consumo? Con los datos actuales, la $\mathrm{T}$ vale 0.041589. Normalmente, en lugar de hablar de eficiencia tecnológica, se habla de Factor X, que es el factor de eficiencia y que es la inversa de $\mathrm{T}$, que en nuestro problema ejemplo tiene un valor de 24 . ¿ Y qué indica esto? Que necesitamos una tecnología 24 veces más eficiente que la actual. Como los datos son proyecciones futuras, vamos a dar un margen amplio y digamos que la tecnología que necesitamos en 2050 debe ser entre 20 y 30 veces más eficiente. ¿Qué quiere decir un factor X de 20 o un factor $\mathrm{X}$ de 30 ? Para que veamos la magnitud de la tragedia, por ejemplo, un factor $\mathrm{X}$ igual a dos significa ser un $50 \%$ más eficientes en cuatro vectores: en consumo de recursos, en consumo de energía, en generación de residuos y en generación de contaminación para producir el mismo producto o servicio. Un factor X igual a 20 significa $95 \%$. Y 30 es un 
$96,7 \%$. Es decir, producir los mismos servicios, siendo un $95 \%$ más eficiente en estos 4 vectores. ¿Esto va a pasar? La respuesta es no. Los saltos de innovación tecnológica no van en esa dirección. Y siempre que hay un salto tecnológico de innovación, suele tocar solo uno de estos vectores, o recursos o energía o contaminación o residuos. Pero no de manera global. Y aquí es donde está el problema.

¿Qué es lo que hace falta? Evidentemente el tema de eficiencia, que la podemos definir: cómo hacer las cosas adecuadamente. Pero también hay que ser eficaz, es decir, hacer las cosas que se tienen que hacer. Y hay otro concepto muy importante que tenemos que ir incorporando a nuestro vocabulario, que es el concepto de suficiencia. Y que no es nuevo. Ya Gandhi lo decía: «Hay suficiente en el mundo para las necesidades humanas pero no para sus deseos». Y la suficiencia lo que pretende es reducir los impactos, reduciendo la demanda de productos. Porque nuevamente lo que hacemos es comprar productos para cubrir necesidades que difícilmente se pueden cubrir con ciertos productos ( $\mathrm{Al}$ menos en la sociedad occidental). Sociedades ya ricas económicamente

Pasemos a introducir el reto en el ámbito social. Existen varios índices, uno muy interesante es el índice del planeta feliz. Utiliza dos factores: el factor social y el factor ambiental. El factor social lo mide con lo que llama «Los años de vida feliz». Se mide con cuán feliz se siente la gente y cuántos años vive; lo que se llama la escalera de la vida. El factor ambiental lo mide con la huella ecológica. Veamos la situación de los países en estos indicadores. Empezamos con la huella ecológica (figura 15):

Figura 15

Huella ecológica per cápita

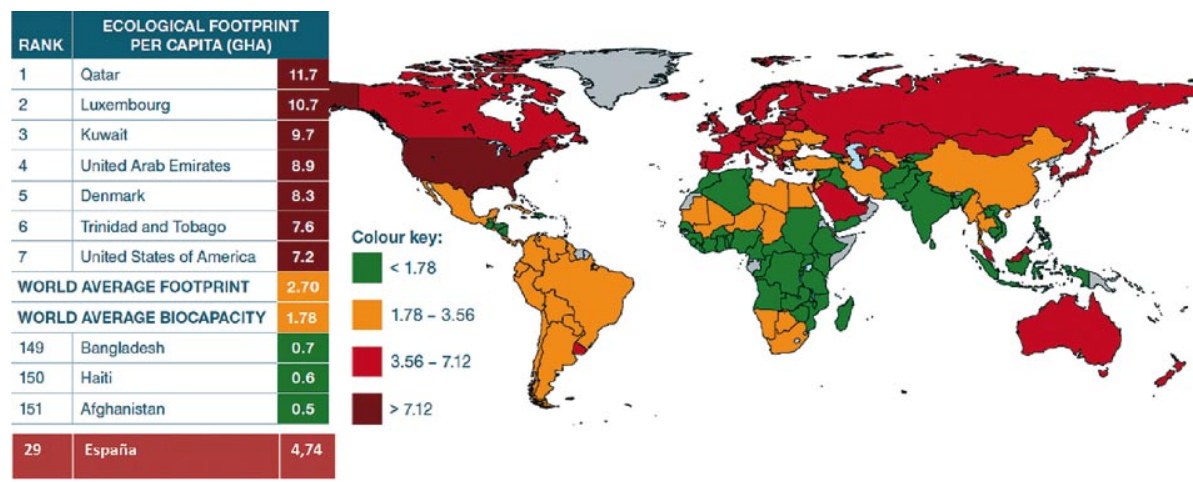


Estos son datos del 2013. España está en 4,74. Los españoles de promedio gastamos dos planetas y medio; si todo el mundo consumiese como nosotros.

Otro indicador es cuán feliz se siente la gente figura 16. Los más felices actualmente son los daneses. Luego está Canadá, está Noruega, Suiza, Suecia. Como podéis ver hay una relación entre huella ecológica y felicidad para ser feliz todavía tengo que consumir.

Figura 16

Valores globales del indicador de bienestar experimentado

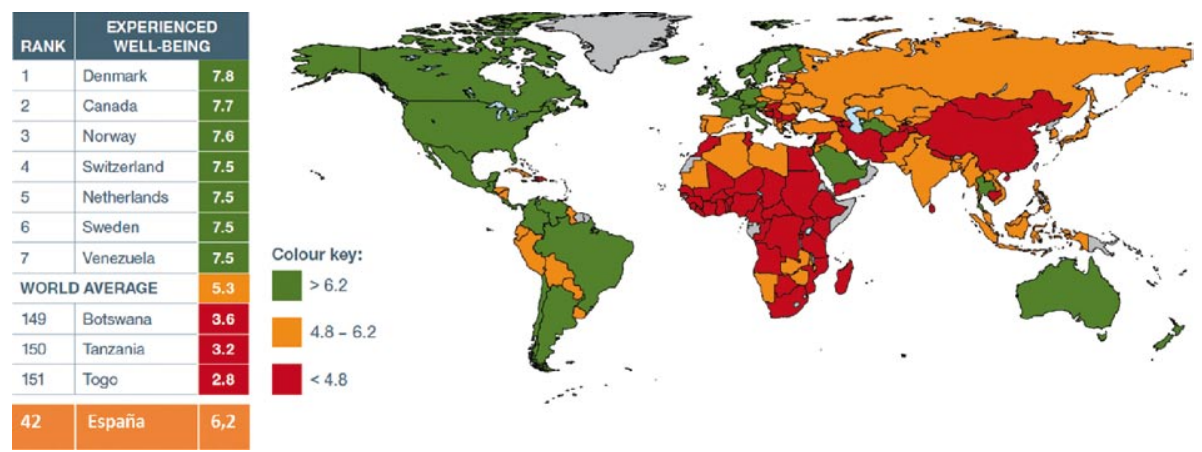

Figura 17

Índice de planeta feliz

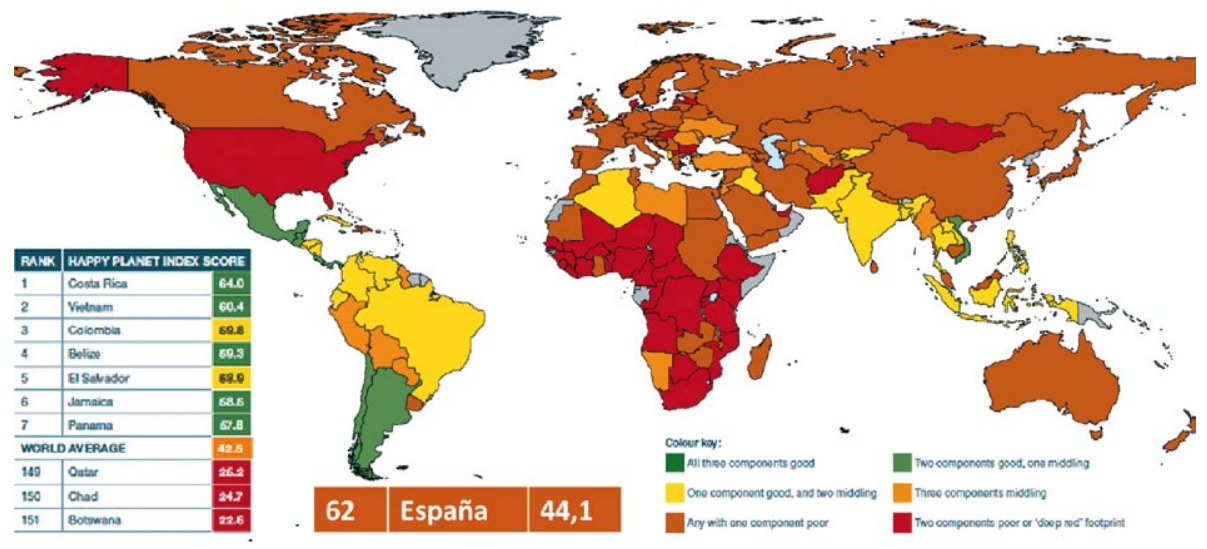

Si juntamos los tres indicadores en el índice de planeta feliz, se observa que no hay ningún país que cumpla con los tres indicadores, ninguno que esté por 
debajo de los límites. Los que están mejor, en este caso son Costa Rica, Vietnam, que no son países realmente occidentales porque tienen poca huella ecológica. La gente vive una vida más o menos larga y se siente feliz. El modelo occidental igual no funciona tan bien. España está en la posición 62.

Volviendo al embudo, aumentamos demanda, disminuyen recursos, vamos chocando con las paredes, hemos de cambiar para no colapsarnos. ¿Qué hace falta para cambiar este paradigma? ¿En qué dimensiones? Básicamente hablamos de 3 niveles: el marco, el nivel de innovación y los actores que se tienen que implicar (figura 18).

Respecto al marco hemos de contemplar tres dimensiones: primero el marco cultural, cuáles son los valores y principios que nos rigen. En principio, han de ser que justifiquen las necesidades, no los deseos. Aquí es donde entra el concepto de suficiencia, si no conseguimos cambiar el marco cultural, es difícil. Segundo, el marco estructural y aquí entran los de relaciones laborales que serían institución institucional legal y económica. Y tercero el marco tecnológico que es medios para satisfacer estas necesidades, definimos qué necesidades son y qué medios vamos a poner para conseguirlas.

Figura 18

Dimensiones del cambio de paradigma

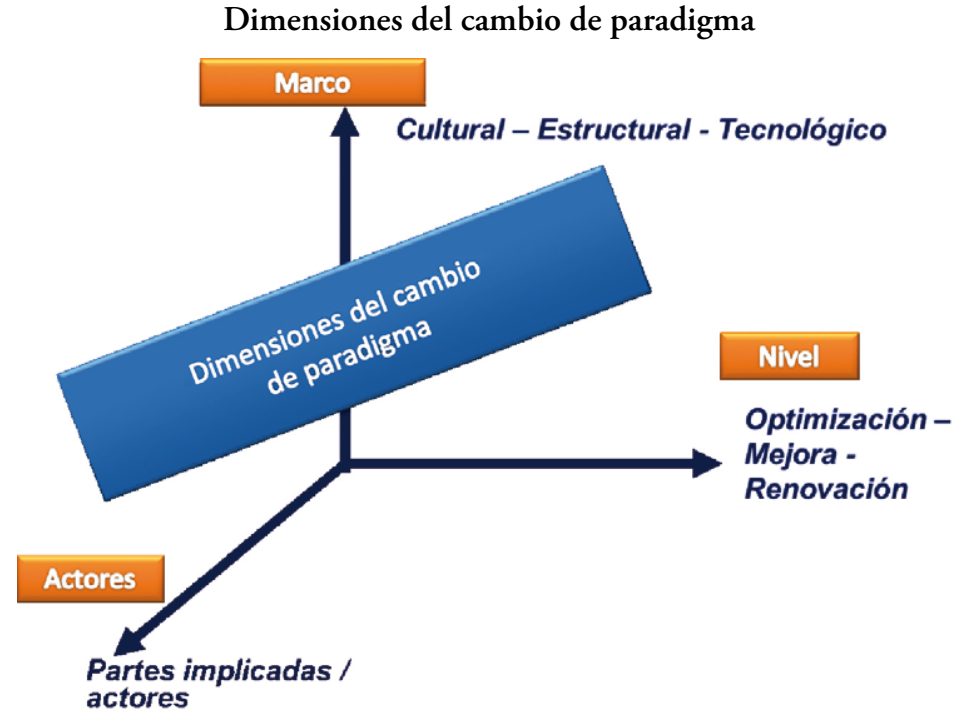

El cambio en el nivel de innovación que básicamente consiste en tres saltos de innovación: primero, el uso óptimo. Esto sería, si usamos el ejemplo de la innovación en el sector del automóvil, lo que ha pasado en los últimos 20 ańos, 
en los que los coches cada vez han sido más eficientes en consumo de combustible por kilometro. Segundo, la mejora en procesos y productos. Pasamos del coche de combustión interna al coche eléctrico. Seguimos moviéndonos en coche, pero ahora en un coche eléctrico que es más eficiente, produce menos emisiones de $\mathrm{CO}_{2}$ por kilómetro; $\mathrm{Y}$ tercero, la renovación basada en necesidades y funciones. ¿Necesito moverme? ¿Cuándo necesito moverme? ¿Y cómo me puedo mover? Vamos a rediseñar por qué me muevo. Y aquí, por ejemplo, a nivel de relaciones laborales, está todo el tema de teletrabajo.

En relación a los actores, se han de tener en cuenta todos los actores implicados o afectados por cualquier decisión socio-política-tecnológica.

\section{Nuevo paradigma educativo}

Teniendo en cuenta estos tres cambios de paradigma: la innovación tecnológica que hace falta, basado en necesidades y funciones, el cambio de estructura, de marco, y el cambio en el rol a jugar por los actores. ¿Qué representa esto para el sistema educativo? Teniendo en cuenta que, en principio, el objetivo es un futuro sostenible. Se plantea el cambio en tres niveles (figura 19).

Figura 19

Niveles del sistema educativo y cambio hacia la Educación Sostenible

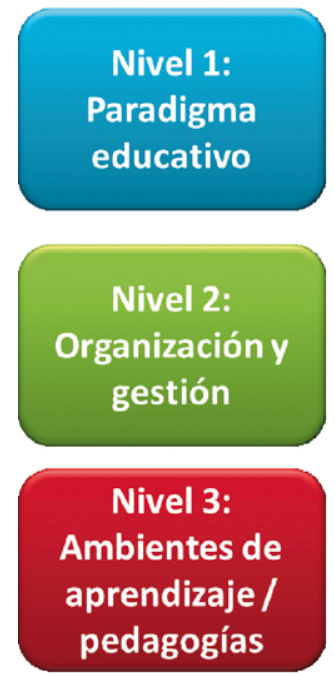

Transformación de los valores Visión y misión

Comunidad

Gestión

Medida y Evaluación Diseño y desarrollo del currículo

Visión del proceso enseñanza/aprendizaje Visión del estudiante Estrategias y estilos de enseñanza aprendizaje 
El primero es el paradigma educativo, que es la transformación de los valores del sistema propio educativo. ¿Cuál es la visión y la misión de la Universidad en este caso? ¿Es formar solo para formar para el mercado laboral? ¿O es para formar titulados críticos con el sistema que puedan mejorar el cambio, puedan mejorar sistemas, formar agentes de cambio?

A partir de ahí, si tenemos una misión que va alineada con la sostenibilidad, ¿cómo nos organizamos y gestionamos? ¿Cómo está organizada la comunidad universitaria concorde a la gestión, evaluación, diseño y desarrollo del currículum?

Y finalmente, cómo enseñamos, cómo visualizamos el proceso de enseñanzaaprendizaje, cómo vemos al estudiante que tenemos delante de nosotros, cuáles son las estrategias. Es decir, hace falta lo que decíamos antes: cambios en las estructuras, en los marcos y en los actores, que sería un cambio: paradigma, objetivo que tenemos como sistema educativo, las políticas que puedan llevarnos a este objetivo y cuál es la práctica.

\section{Conclusiones}

La situación actual es insostenible a medio plazo. Se requiere un cambio de paradigma en nuestra sociedad y con ello un cambio de paradigma en el sistema educativo.

Es imprescindible cuestionarse para qué y para quién educamos y, teniendo en cuenta los retos de sostenibilidad a que se enfrenta nuestra sociedad, redefinir un nuevo paradigma para la educación con una visión sostenible. Hace falta introducir conceptos, efectividad y suficiencia. Se requieren procesos transdisciplinares, en todo el ámbito académico, entendiendo por transdisciplinaridad el hecho de involucrar a la sociedad en lo que sería generación de nuevo conocimiento. Y en la misma educación hace falta un cambio de paradigma, de objetivos, de políticas. 